\title{
ESTIMANDO LA DISPOSICIÓN A PAGAR POR LA CONSERVACIÓN DE LOS PASTIZALES ALTO ANDINOS
}

\section{ESTIMATING THE WILLINGNESS TO PAY FOR THE CONSERVATION OF ANDEAN RANGELANDS}

\author{
Cecilio A. Barrantes ${ }^{1}$ y Enrique R. Flores ${ }^{2}$
}

\begin{abstract}
Resumen
Se realizó un estudio para estimar la disponibilidad a pagar (DAP) para la implementación de un programa de conservación y mejoramiento de pastizales (PCMP) en la Región Pasco, mediante el método de valoración contingente. Para estimar la DAP se aplicó una encuesta preliminar de tipo abierta a 30 pobladores y otra cerrada en formato binario a otros 105. Los resultados de la encuesta preliminar abierta permitieron definir siete vectores de pagos o BIDs (S/. 1, 5, 10, 20, 25, 30 y 40), a partir de los cuales se aplicó una encuesta cerrada a razón de 15 personas por BID. Los resultados de la encuesta cerrada así obtenida se vaciaron en el programa NLOGIT 3.0 para su corrida utilizando una distribución LOGIT, en tres etapas. La primera con todas las variables de la encuesta, la segunda con las variables socioeconómicas y la tercera con el ingreso familiar mensual. Los outputs de las tres etapas produjeron resultados similares en los signos, negativo para BID y positivo para los ingresos familiares. La DAP fue S/. 3.94/familia/mes, revelando que la Región Pasco podría recaudar anualmente aproximadamente 1.95 millones de soles para la implementación del PCMP.
\end{abstract}

Palabras clave: disponibilidad a pagar, valoración contingente, pastizales alto andinos.

\begin{abstract}
A study was conducted to estimate willingness to pay (WTP) for the implementation of the Range Conservation and Improvement Program (RCIP) in the Pasco Region, using a contingent valuation method. To estimate the WTP we conducted a preliminary open survey to 30 respondents and a closed binary format to other 105 respondents. The open survey allowed to define seven vectors of payment or BIDs (S/. 1, 5, 10, 20, 25, 30 and 40) to which we applied a closed surveys 15 for each BID. The results of the closed survey were run on NLOGIT 3.0, using a LOGIT distributions in three stages, the first with all the variables of the survey, the second with the socioeconomic variables and third with monthly family income as a unique variable. The outputs of the three stage running process were similar in terms of signs, BID (negative) and monthly family income (positive). The WTP was S/. 3.94/family/month, revealing that the regional government of Pasco could have S/. 1.95 millions of soles available to invest in a RCIP.
\end{abstract}

Key words: willingness to pay, contingent valuation, andean rangelands.

\section{Introducción.}

Las praderas naturales alto andinas han sido consideradas el segundo ecosistema en importancia ecológica después de los bosques tropicales no solo por su extensión, más de 10.5 millones de hectáreas, sino también por su capacidad para brindar servicios ambientales claves a la sociedad, como la regulación del ciclo hídrico, protección de cuencas y secuestro de carbono (FAO, 2009) y contribuir significativamente a la seguridad alimentaria, si se tiene en cuenta que más del $80 \%$ de la población ganadera del país utiliza este recurso natural renovable como fuente principal de alimento (Flores, 1996; Recharte et al., 2002). Estudios recientes revelan que la producción de carne y leche de 4.5 millones de vacunos, 13.6 millones de ovinos, 4.2 millones de alpacas y 1.2 millones de llamas, que usan los pastizales alto andinos, estarían en capacidad de satisfacer $47 \%$ del consumo de carne y $24 \%$ del consumo de leche de la población a nivel nacional (Flores, 2012).

El aprovechamiento integral de las potencialidades ecológicas y económicas que ofrecen los pastizales, sin embargo, no está libre de limitaciones, la pobreza, el sobre pastoreo y mal manejo son fenómenos comunes en los andes, determinando el deterioro del potencial ecológico y económico de estos ecosistemas (Huerta, 2002). Análisis del estatus ecológico de los pastizales revelan que más del $60 \%$ de los pastizales están en condición pobre y que por lo menos el $50 \%$ estarían en un franco proceso de deterioro o tendencia negativa, proceso que genera pérdidas económicas al estado nacional del orden de los 600 millones de 
dólares anuales (Flores, 2011). En consecuencia, es necesario desarrollar políticas orientadas a revertir este proceso, lo cual es muy importante, más aun si se tiene en cuenta que en la medida que la agricultura desarrolla y el uso del agua y la tierra se vuelve más intenso, el cambio climático acentúa la probabilidad de ocurrencia de eventos extremos (Martínez et al., 2006; Yahdjian \& Sala, 2011); de modo que su impacto en la integridad de los ecosistemas naturales podría tornarse muy intenso, reduciendo su capacidad del ecosistema para producir alimentos (FAO, 2011).

En el Perú los recursos son escasos y se carece de estimaciones del valor económico de los bienes y servicios ambientales que provee los ecosistemas de pastizal. El desarrollo de políticas de conservación y mejora de los recursos naturales requiere de la asignación de recursos económicos y normas por parte de los decisores políticos para regular su uso y conservación y el compromiso tanto de los usuarios de las tierras de pastoreo como de la sociedad en su conjunto (Flores, 2011). El presente estudio fue diseñado con el objetivo de valorar económicamente el valor de existencia de los pastizales por el método de valoración contingente, preguntándoles a los pobladores cuanto estarían dispuestos a pagar por la implementación de un programa de conservación y mejoramiento de praderas asegurando así la continuidad en el suministro de los bienes y servicios ambientales que ofrecen los pastizales (Sarmiento \& Rodríguez, 2005; Villena \& Lafuente, 2012).

\section{Materiales y métodos.}

El estudio de valoración contingente se realizó en la ciudad de Cerro de Pasco entre Agosto y Setiembre del 2011, a través de una entrevista a 135 pobladores, entre beneficiarios directos e indirectos de los bienes y servicios que ofrece este ecosistema de montaña. Los pastizales alto andinos del país están conformados por una combinación de pajonales, césped de puna, bofedales y arbustales, que cubren una extensión de 507955.6 ha la mayoría en condición pobre y franco proceso de deterioro debido al sobrepastoreo y mal manejo (Zarria \& Flores, 2012). Un breve análisis de las características socioeconómicas de la población basadas en encuestas recientes revela que en la ciudad de Cerro de Pasco convergen pobladores, en su mayoría usuarios directos de los pastizales, que habitan en la zona alto andina de la provincia de Pasco y Daniel Alcides Carrión, una zona eminentemente ganadera. Estas familias están compuestas en promedio por 5.8 integrantes, con un ingreso familiar mensual de 786 nuevos soles, un tiempo promedio de estudio de 13 años, una edad promedio 36 años, donde el $44 \%$ tienen estudios superiores y $66 \%$ tienen empleo; es decir, se trata de una población de bajos ingresos, familias numerosas en estado de pobreza, que no han terminado su educación superior.
Para realizar la valoración contingente se usó dos tipos de encuesta, una abierta y otra cerrada, para estimar la disponibilidad a pagar de los pobladores de Pasco por un programa de conservación $\mathrm{y}$ mejoramiento de pastizales (PCMP), tomando en cuenta que en países en vías de desarrollo se recomienda elegir DAP más conservadora de las que arrojan las diferentes metodologías disponibles y que además esté cercana con las medidas de tendencia central como la media, mediana o moda. Para nuestro estudio la DAP se comparó con la mediana, con la finalidad de tener un valor referencial que indican el $50 \%$ de las familias, además, que no se ve afectado por valores extremos que arrojan las encuestas (Whitehead, 1990; Farber et al., 2002; Riera et al., 2005; Han et al., 2011; Villena \& Lafuente, 2012).

En la primera etapa se utilizó una encuesta de carácter abierto compuesta de 9 preguntas, que se aplicó a 30 personas elegidas al azar sin distinción de sexo y con la única restricción de elección que fueran mayores de edad y contribuyan económicamente a la canasta básica familiar. Las primeras ocho preguntas fueron de carácter general y tuvieron que ver con el conocimiento del ecosistema pastizal, comunidades campesinas y servicios ambientales, mientras que la novena consistió en preguntar ¿Cuánto estarían dispuestos usted y su familia a pagar mensualmente como incremento en su recibo de luz, para la implementación del PCMP, evitando así que los pastizales sigan destruyéndose y conservarlos para poder seguir visitándolos y beneficiándonos de sus servicios ambientales? Con esta encuesta inicial se pudo medir el nivel de conocimiento del ecosistema pastizal, como este influye en su estilo de vida y obtener siete vectores de pagos o BIDs, que sirvieron posteriormente para diseñar la encuesta cerrada de formato binario (Riera et al., 2005; Villena \& Lafuente, 2012).

En una segunda etapa se aplicó una encuesta cerrada de formato binario a 15 pobladores por BID estimado en la primera etapa, haciendo un total de 105 encuestas lo cual permitió estimar la DAP de los pobladores para la implementación del PCMP con nivel de confianza del $95 \%$ y una precisión del 10\% del promedio (Chaves, 2008; Villena \& Lafuente, 2012). La encuesta tenia quince preguntas de respuesta dicotómica ( $\mathrm{Si}$ y No), ocho fueron similares a las de la encuesta abierta y la diferencia siete diferentes, seis fueron de carácter social (ingreso familiar mensual, estado civil, edad, sexo, nivel educativo y miembros de familia) y la última económica. Se les pedía responder si aceptaban o rechazaban el pago mensual para la implementación del PCMP en la Región Pasco (Whitehead, 1990; Riera et al., 2005; Villena \& Lafuente, 2012).

Para estimar la DAP, la información de las encuestas fueron vaciadas en el programa NLOGIT 3.0, y corrida tres veces, en la primera se estableció las 
magnitudes de los signos y coeficientes de regresión para cada uno de las variables incorporadas al modelo LOGIT, en la segunda corrida y solo con las variables sociales se estimó los coeficientes de regresión, pendientes de los coeficientes y la DAP, y en la tercera se determinó la DAP a partir del ingreso familiar mensual. En todas las corridas se estimó la bondad de ajuste como el $\mathrm{R}^{2} \mathrm{Mc}$ Fadden y Chi Cuadrado, lo que permitió establecer la capacidad de predicción correcta del modelo (Whitehead, 1990; Loomis et al., 2008; Han et al., 2011; Villena \& Lafuente, 2012).

\section{Resultados y discusión.}

El análisis de los resultados de la encuesta preliminar abierta reveló que la mayoría de los pobladores consideran que los pastizales son importantes para el desarrollo de sus actividades sociales y económicas, y que están bajo amenaza por lo que deberían protegerse. Cuando se les preguntó cuánto estarían dispuestos a pagar mensualmente en el recibo de luz ellos y sus familias, para implementar un programa para mantener a los ecosistemas saludables $\mathrm{y}$ que sigan brindando servicios ambientales a la población, el $63 \%$ contestó afirmativamente en un rango de valores que variaba entre 1 a 40 soles, sugiriendo que los vectores de pago (BID) más adecuados para definir la función de pago serian: 1, 5, 10, 20, 25, 30 y 40 soles (ILC, 2011; Han et al., 2011; Villena \& Lafuente, 2012).

La sistematización de la encuesta cerrada de formato binario elaborada a partir de los BIDs se muestra en el Tabla 1, revelan que más del $60 \%$ de los encuestados afirmaron que conocían los pastizales y los consideraban importantes, confirmando las tendencias obtenidas en la fase abierta de la encuesta en relación a que el pastizal es un espacio importante que provee oportunidades para actividades de esparcimiento al aire libre, opciones económicas y que se encuentra bajo amenaza. La encuesta también indica que muy pocos de los encuestados $1 \%$ conocen que es un servicio ambiental así como las implicancias económicas y ecológicas que podría tener implementar un PCMP, sin embargo la mayoría considera de extrema importancia la conservación del ecosistema sugiriendo que el concepto de valor de los servicios ambientales todavía no ha sido internalizado por la población y que se requiere mayor información e investigación sobre la capacidad de abastecimiento de servicios y valor económico de los pastizales alto andinos. Encuestas a rancheros norteamericanos revelan que más del $70 \%$ tenían muy poco o ningún conocimiento acerca de los servicios ambientales y un $63 \%$ de ellos puntos de vista negativos del secuestro de carbono. Solamente el $4 \%$ de los encuestados mostraron una disponibilidad incondicional a participar en programas de secuestro de carbono pero el $71 \%$ manifestó que participarían eventualmente dependiendo de nueva información (Ma \& Coppock, 2012).

Tabla 1: Resultados de la Encuesta Cerrada de Formato Binario.

\begin{tabular}{|c|c|c|c|}
\hline \multirow[t]{2}{*}{$\mathrm{N}^{\circ}$} & \multirow{2}{*}{ Pregunta } & \multicolumn{2}{|c|}{$\begin{array}{l}\text { Respuesta } \\
(\%)\end{array}$} \\
\hline & & $\mathrm{Si}$ & No \\
\hline 1 & $\begin{array}{l}\text { ¿Acostumbra a realizar actividades al } \\
\text { aire libre como paseos, pesca u otros } \\
\text { en lugares naturales como bosques, } \\
\text { ríos, lagos u otros similares? }\end{array}$ & 96.2 & 3.8 \\
\hline 2 & $\begin{array}{l}\text { ¿Conoce usted los pastizales alto } \\
\text { andinos? }\end{array}$ & 64.8 & 35.2 \\
\hline 3 & $\begin{array}{l}\text { ¿Es para usted importante los } \\
\text { pastizales alto andinos? }\end{array}$ & 99.0 & 1.0 \\
\hline 4 & $\begin{array}{l}\text { ¿Conoce Ud. de que se trata un } \\
\text { "programa de mejoramiento y } \\
\text { conservación de pastizales alto } \\
\text { andinos"? }\end{array}$ & 4.8 & 95.2 \\
\hline 5 & $\begin{array}{l}\text { ¿Usted ha visitado los pastizales alto } \\
\text { andinos de la región Pasco? }\end{array}$ & 91.4 & 8.6 \\
\hline 6 & $\begin{array}{l}\text { ¿Conoce usted que es un servicio } \\
\text { ambiental? }\end{array}$ & 1.0 & 99.0 \\
\hline 7 & $\begin{array}{l}\text { ¿En su opinión cree usted que los } \\
\text { pastizales alto andinos de la región } \\
\text { Pasco; está bajo amenaza? }\end{array}$ & 53.3 & 46.7 \\
\hline 8 & $\begin{array}{l}\text { ¿En su opinión, es importante para } \\
\text { usted que los pastizales alto andinos } \\
\text { deban protegerse? }\end{array}$ & 98.1 & 1.9 \\
\hline
\end{tabular}

Cuando se les preguntó sobre su disposición a pagar por un determinado BID mensualmente en el recibo de energía eléctrica para la implementación de un PCMP se encontró que la proporción de respuestas positivas disminuía cuando el vector de pago aumentaba (Tabla 2) aspecto que consistente con la teoría económica de la demanda por un bien o servicio; es decir, a menor vector de pago mayor la disposición a pagar (Farber et al., 2002; Riera et al., 2005). Un análisis de la relación funcional entre el porcentaje de familias que estaban a dispuesta a pagar y el vector de pago $\left(\mathrm{Y}=-22.2 \mathrm{Ln}(\mathrm{X})+79.356, \mathrm{R}^{2}=\right.$ $0.7268)$ confirmó la relación negativa entre el valor del vector de pago y la disposición a pagar. Los resultados también revelaron que un cincuenta por ciento de las familias estarían dispuestas a pagar 3.75 nuevos soles por mes, un valor cercano a aquel

Tabla 2: Proporción de Respuestas Positivas a los BID.

\begin{tabular}{ccccc}
\hline \multirow{2}{*}{$\begin{array}{c}\text { BID } \\
\text { (S/.) }\end{array}$} & Positivo & Negativo & Total & $\begin{array}{c}\text { Proporción } \\
\text { Respuesta } \\
\text { Positiva }\end{array}$ \\
\hline 1 & 15 & 0 & 15 & 1,00 \\
5 & 2 & 13 & 15 & 0,13 \\
10 & 2 & 13 & 15 & 0,13 \\
20 & 2 & 13 & 15 & 0,13 \\
25 & 2 & 13 & 15 & 0,13 \\
30 & 3 & 12 & 15 & 0,20 \\
40 & 0 & 15 & 15 & 0,00 \\
\hline
\end{tabular}


predicho por el modelo LOGIT ,3.94 soles mensuales, monto finalmente escogido por representar un valor ajustado en función del ingreso mensual, estado civil, edad y sexo.

Los resultados muestran también que las dos principales razones por las cuales muchos de los encuestados no estarían dispuestos a pagar por el programa, tendrían que ver que sus recursos económicos son escasos (56\%) y la percepción de que deberían ser terceros (minería, gobierno regional) (38\%) quienes consideran deberían asumir un rol protagónico en la conservación de los recursos naturales. Entre otros factores que habrían determinado su negativa a pagar estarían su desconfianza sobre el destino de los montos recaudados (Tabla 3 ).

Tabla 3: Motivo de la Negativa a Pagar

\begin{tabular}{lr}
\hline \multicolumn{1}{c}{ Motivo } & $\begin{array}{r}\text { Porcentaje } \\
(\%)\end{array}$ \\
\hline $\begin{array}{l}\text { No tengo los medios económicos } \\
\text { suficientes para pagar }\end{array}$ & 55.6 \\
$\begin{array}{l}\text { La minería debe pagar por esto } \\
\text { El gobierno debe pagar por esto }\end{array}$ & 29.2 \\
$\begin{array}{l}\text { Creo que el fondo será mal } \\
\text { utilizado }\end{array}$ & 5.6 \\
$\begin{array}{l}\text { Pienso que este problema no es } \\
\text { prioritario }\end{array}$ & 1.2 \\
\hline
\end{tabular}

Los resultados de la primera corrida con todas las variables en términos de valor de los coeficientes, error estándar y significancia estadística se muestra en el Tabla 4, observándose que el coeficiente de regresión de la BID para el modelo LOGIT fue negativa $(\mathrm{p}<0.01)$ mientras que el coeficiente para el ingreso mensual fue positiva pero no significativa $(\mathrm{p}>$ 0.05 ). Lo cual confirma que a mayor el valor de un bien la disponibilidad a pagar por el mismo es menor, en tanto que a mayor ingreso familiar la tendencia es a pagar más por el mismo. Analizando el comportamiento de las otras variables se encuentra que el ecosistema forma parte de su estilo de vida (paseos, importancia, considera amenazado, debe ser protegido los pastizales) y si el encuestado tienen más años de estudio la DAP aumenta, mientras los que son de familia numerosa y casados la DAP disminuye.

En referencia a la bondad de ajuste del modelo, los valores de la $\mathrm{R}^{2}$ Mc Fadden $\left(\mathrm{R}^{2}=0.2205\right)$ para una DAP de S/. 3.94, basada en variables en socioeconómicas, se observó que este fue superior a los encontrados en estudios destinados a estimar el beneficio marginal derivado de la quema contralada en programas de reducción de combustible para prevenir quemas fortuitas $\left(\mathrm{R}^{2} \mathrm{Mc}\right.$ Fadden $<0.0779$, Loomis et al., 2008) y otros en que se utilizo Chi - cuadrado como indicador de la relación significativa entre el BID y el proporción de familias dispuestas a pagar por determinado BID bosques de algarrobo (Villena \&
Lafuente, 2012), así como humedales (Whitehead, 1990) y en reservas naturales (Han et al., 2011).

Tabla 4: Coeficientes de Todas las Variables del Modelo Logístico.

\begin{tabular}{|c|c|c|c|}
\hline Variables & LOGIT & Error Estándar & P value \\
\hline Constante & -57.6714 & $0.518583 \mathrm{D}+07$ & 1.0000 \\
\hline BID & -0.1168 & 0.02866281 & 0.0000 \\
\hline $\begin{array}{l}\text { Ingreso Familiar } \\
\text { Mensual }\end{array}$ & 0.0006 & 0.00088469 & 0.4700 \\
\hline Nivel Educativo & 0.1196 & 0.08988032 & 0.1832 \\
\hline $\begin{array}{l}\text { Miembros } \\
\text { Familia }\end{array}$ & -0.1315 & 0.10306603 & 0.2021 \\
\hline $\begin{array}{l}\text { Paseos } \\
\text { Pastizales }\end{array}$ & 30.0868 & $0.243447 \mathrm{D}+07$ & 1.0000 \\
\hline $\begin{array}{l}\text { Conoce } \\
\text { Pastizales }\end{array}$ & -0.3160 & 0.62668621 & 0.6141 \\
\hline $\begin{array}{l}\text { Importante } \\
\text { Pastizales }\end{array}$ & 0.5959 & $0.497046 \mathrm{D}+07$ & 1.0000 \\
\hline Conoce PCMP & -0.0779 & 1.33196991 & 0.9533 \\
\hline $\begin{array}{l}\text { Visita Pastizales } \\
\text { Conoce }\end{array}$ & -1.0447 & 1.22576537 & 0.3940 \\
\hline $\begin{array}{l}\text { Servicios } \\
\text { Ambientales }\end{array}$ & -31.8662 & $0.435460 \mathrm{D}+07$ & 1.0000 \\
\hline $\begin{array}{l}\text { Pastizales } \\
\text { Amenazado }\end{array}$ & 0.3779 & 0.62836729 & 0.5475 \\
\hline $\begin{array}{l}\text { Pastizales } \\
\text { Protegerse }\end{array}$ & 27.1687 & $0.275898 \mathrm{D}+07$ & 1.0000 \\
\hline Estado Civil & -0.6134 & 0.68896430 & 0.3733 \\
\hline Edad & 0.0111 & 0.03255910 & 0.7336 \\
\hline Sexo & 0.3355 & 0.64066592 & 0.6005 \\
\hline $\begin{array}{l}\text { Tamaño de } \\
\text { muestra }\end{array}$ & & & 105 \\
\hline $\begin{array}{l}\text { Grados de } \\
\text { libertad }\end{array}$ & & & 15 \\
\hline $\mathrm{R}^{2} \mathrm{Mc}$ Fadden & & & 0.29911 \\
\hline Chi Cuadrado & & & $35.1573 *$ \\
\hline $\begin{array}{l}\text { Predicción } \\
\text { Correcta }(\%)\end{array}$ & & & 83.81 \\
\hline
\end{tabular}

En la segunda corrida que incluía solo las variables socioeconómicas, tal como lo muestra el Tabla 5, la BID mostró un signo negativo mientras que el ingreso familiar mensual un signo positivo al igual que en la primera corrida, confirmando las tendencias anteriores. Los resultados también muestran que cuando el encuestado es casado su DAP disminuye, lo cual es previsible desde que personas casadas usualmente experimentan mayores niveles de gastos. La extensión de este análisis a las variables de edad y sexo revela que estos dos factores también influyen en la magnitud de la DAP, es decir, tanto los jóvenes como los individuos de sexo masculino muestran una mayor disposición a contribuir al PCMP (Whitehead, 1990; Han et al., 2011). 
Tabla 5: Coeficientes de las Variables Socioeconómicas del Modelo Logit.

\begin{tabular}{|c|c|c|c|c|}
\hline Variables & LOGIT & $\begin{array}{l}\text { Error } \\
\text { Estándar }\end{array}$ & $\mathrm{P}$ value & $\operatorname{Exp}(\beta)$ \\
\hline Constante & 0.6980 & 1.06331570 & 0.5115 & 2.0097 \\
\hline BID & -0.1050 & 0.02587262 & 0.0000 & 0.9003 \\
\hline Ingreso & & & & \\
\hline Familiar & 0.0009 & 0.00068905 & 0.2059 & 1.0009 \\
\hline Civil & -0.4431 & 0.58837288 & .4514 & 0.6420 \\
\hline Casado & & & & \\
\hline Edad & -0.0205 & 0.02580857 & 0.4274 & 0.9797 \\
\hline $\begin{array}{l}\text { Sexo } \\
\text { Masculino }\end{array}$ & 0.1192 & 0.58784667 & 0.8393 & 1.1266 \\
\hline $\begin{array}{l}\text { Tamaño } \\
\text { de } \\
\text { muestra }\end{array}$ & & & & 105 \\
\hline $\begin{array}{l}\text { Grados de } \\
\text { libertad }\end{array}$ & & & & 5 \\
\hline $\begin{array}{l}\mathrm{R}^{2} \quad \mathrm{Mc} \\
\text { Fadden }\end{array}$ & & & & 0.22052 \\
\hline Chi & & & & $501040 *$ \\
\hline Cuadrado & & & & 5.91940 \\
\hline Predicción & & & & \\
\hline $\begin{array}{l}\text { Correcta } \\
(\%)\end{array}$ & & & & 81.905 \\
\hline DAP (S/.) & & & & 3.9378 \\
\hline
\end{tabular}

El valor de la DAP a partir de las variables socioeconómicas obtenida por el modelo LOGIT fue de S/ 3.94 a pagar en el recibo mensual de luz son consistentes con los datos obtenidos en la primera corrida, además que tiene un Chi - cuadrado significativo y un nivel de predicción correcta mayor a $80 \%$. Al hacer el análisis de los efectos marginales, es decir, cuanto varia la DAP cuando una variable explicadora cambia en una unidad y las demás permanecen constantes (Riera et al., 2005; Chaves, 2008), se observó que si el ingreso familiar aumenta en un nuevo sol (S/1.00) la DAP resultante aumentaría también en 1.00 nuevo sol, es decir a mayor nivel de ingreso mayor disposición a pagar por el PCMP. En cambio, si la BID aumentaría en un nuevo sol (S/1.00), la DAP disminuiría en 0.90 nuevos soles, revelando que a mayor valor de la BID menor disposición a pagar por el PCMP. Con respecto a las variables socioeconómicas (estado civil, edad y sexo), cuando los encuestados son casados, el valor de la DAP disminuye en S/ 0.64, cuando la edad incrementa en un año, la DAP disminuye en 0.98 nuevos soles y cuando las personas son del sexo masculino la DAP aumentaría en 1.13 nuevos soles

En la tercera corrida cuando se utilizó solo la variable ingreso familiar mensual para la determinación de la DAP, se observo al igual que en las dos corridas anteriores que el coeficiente de la BID fue negativa y el de ingreso familiar positiva. La DAP en esta última corrida fue de $\mathrm{S} / .4 .14$ adicionales sobre el consumo de energía eléctrica mensual para la implementación del PCMP en la Región Pasco (Tabla $6)$.

Con respecto a la bondad de ajuste del modelo observado en esta última corrida se encontró que el valor Chi Cuadrado fue significativo al igual que el $\mathrm{R}^{2}$ Mc Fadden que fue ligeramente inferior en comparación con la corrida anterior. Un breve análisis de los efectos marginales, revela valores similares a los observados anteriormente así cuando el ingreso familiar aumenta en un nuevo sol (S/1.00) la DAP resultante aumenta en 1.00 nuevo sol, mientras que si la BID aumentaría en un nuevo sol (S/1.00), la DAP disminuiría en 0.90 nuevos soles (Tabla 6). Cabe indicar que la DAP obtenida en esta última corrida, 4.14 fue ligeramente superior a la anterior 3.94 , obtenida en la segunda corrida que consideraba todas las variables socioeconómicas.

En un intento para estimar cuanto se podría recaudar como mínimo para constituir un fondo para la implementación del PCMP para la Región Pasco, se utilizó el valor de la DAP más conservadora y cercana a la mediana (Villena y La Fuente, 2012), esto es la DAP estimada utilizando solo las variables socioeconómicas, ascendiente a S/. 3.94/familia/mes, el cual multiplicado por 41177 familias residentes en las provincias de Pasco y Daniel Alcides Carrión (INEI, 2011), permitiría obtener una recaudación

Tabla 6: Coeficientes de la Variable Ingreso Económico

\begin{tabular}{|c|c|c|c|c|}
\hline Variables & LOGIT & $\begin{array}{l}\text { Error } \\
\text { Estándar }\end{array}$ & P value & $\operatorname{Exp}(\beta)$ \\
\hline Constante & -0.1208 & 0.58195551 & 0.8356 & 0.8862 \\
\hline BID & -0.1063 & 0.02595551 & 0.0000 & 0.8992 \\
\hline $\begin{array}{l}\text { Ingreso } \\
\text { Familiar } \\
\text { Mensual }\end{array}$ & 0.0007 & 0.00063639 & 0.2622 & 1.0007 \\
\hline $\begin{array}{l}\text { Tamaño de } \\
\text { muestra }\end{array}$ & & & & 105 \\
\hline $\begin{array}{l}\text { Grados de } \\
\text { libertad }\end{array}$ & & & & 2 \\
\hline $\begin{array}{l}\mathrm{R}^{2} \quad \mathrm{Mc} \\
\text { Fadden }\end{array}$ & & & & 0.20924 \\
\hline $\begin{array}{l}\text { Chi } \\
\text { Cuadrado } \\
\text { Predicción }\end{array}$ & & & & $24.59326^{*}$ \\
\hline $\begin{array}{l}\text { Correcta } \\
(\%)\end{array}$ & & & & 85.714 \\
\hline $\operatorname{DAP}(\mathrm{S} /)$. & & & & 4.13652 \\
\hline
\end{tabular}

* Significativo $(\alpha=0.05)$

mensual de S/. 162237.38 y una anual de S/. 1946 848.56 anual para el PCMP en Región Pasco. Este fondo podría destinarse para promover la generación de tecnologías de manejo sustentable de pastizales, diseño de políticas de estímulo para la conservación, formación de capacidades técnicas y ejecución de actividades de monitoreo de ecosistemas de pastizales (INRENA 2005; Siffredi, 2009). 


\section{Conclusiones.}

Los resultados indicaron que la mayoría de la población encuestada considera a los pastizales un ecosistema importante pero bajo amenaza que debería conservarse para que continúen existiendo en un adecuado estado de conservación, aspecto por el cual estarían dispuestos a pagar un promedio $\mathrm{S} /$. 3.94/familia/mes, en el recibo de luz destinado a la formación de un fondo para la implementación de un programa de conservación y mejora de pastizales. Esto es muy importante pues significa que se podría recaudar en una acción sin precedentes S/. 1.95 millones de nuevos soles anuales para la implementación de dicho programa. Una vez constituido el fondo y sobre la base de los resultados que se podrían obtener después de una primera etapa piloto y de acción participativa se podrían capturar fondos colaterales de instituciones interesadas en la conservación para la construcción de un programa de conservación y mejora de pastizales de mayor envergadura, que contemple el uso de fondos públicos, privados y de la cooperación internacional. El diseño de un marco institucional y legal para asegurar la sostenibilidad y transparencia del uso del fondo será una tarea a considerar en el futuro para lograr el mantenimiento de la capacidad de los ecosistemas de pastizal para brindar servicios ambientales al conjunto de la sociedad.

\section{Agradecimientos.}

Los autores agradecen al Instituto Latinoamericano de Ciencias por la asesoría en la determinación de la DAP y al Laboratorio de Utilización de Pastizales de la Universidad Nacional Agraria La Molina por el apoyo financiero para el recojo, procesamiento de información y edición de la presente publicación.

\section{Literatura citada.}

Chaves E. 2008. Valoración del agua en la cuenca del rio Tempisque: un ejemplo sobre el método de valoración contingente. UNICIENCIA 22:19 - 31 .

Farber S., Costanza R. \& Wilson M. 2002. Economic and ecological concepts for valuing ecosystem services. En: Ecological Economics. 41: 375 - 392.

Flores E. R. 1996. Reality, limitations and research needs of the peruvian livestock sector. Latin America Regional Livestock Assessment. Workshop Proceedings. IICA SRCP. San José - Costa Rica. April 15-18: 83-99.

Flores E.R. 2011. Importancia de los ecosistemas de puna húmeda para la seguridad hídrica en el contexto del cambio climático global. En: Casa Abierta 2011. Avances y resultados del Proyecto Punas y Agua y del Programa de las Cumbre a la Costa. The Mountain Institute. Lima - Perú.

Flores E.R. 2012. Pastores de puna, cambio climático y seguridad alimentaria. En: Seminario Internacional Seguridad Alimentaria y Economía del Cambio Climático. Lima 6 - 7 septiembre.
Food and Agriculture Organization - FAO. 2011. Climate change, water and food security. FAO Water Reports 36. ISSN 1020-1203. Rome - Italy. 200 pp.

FAO. 2009. Review of evidence on dry lands pastoral systems and climate change: Implications and opportunities for mitigation and adaptation. Roma. 39 pp.

Instituto Latinoamericano de Ciencias - ILC. 2011. Modulo X. Diseño de estudio de valoración contingente. En: Diplomado de Valoración Económica de Ecosistemas con Énfasis en Bosques. Huancayo - Perú.

Han F., Yang Z., Wang H. \& Xu X. 2011. Estimating willingness top ay for environment conservation: a contingent valuation study of Kanas Nature Reserve, Xinjiang, China. En: Environmental Monitoring and Assessment. 180:451 - 459.

Huerta L. 2002. Formulación de herramientas de gestión integral para el manejo sostenible de las praderas altoandinas: estudio de caso en la cabecera de microcuenca Quitaracza - cuenca Santa, Sihuas Ancash. Tesis para optar el título de Ingeniero Zootecnista. Universidad Nacional Agraria La Molina. Lima - Perú. 282 pp.

Instituto Nacional de Estadística e Informática - INEI. 2011. Reporte de población del Perú. Lima - Perú.

Instituto Nacional de Recursos Naturales - INRENA. 2005. Santuario Nacional de Huayllay - Plan Maestro 2005 2010. Ministerio de Agricultura. 132 pp.

Loomis J., Hung L. \& González A. 2008. Willingness - to pay function for two fuel treatments to reduce wildfire acreage burned: a scope test and comparison of white and Hispanic households. En: General Technical Report. PSW - GTR - 227. 168 - 176.

Ma Z. \& Coppock D. 2012. Perceptions of Utah ranchers toward carbon sequestration: policy implications for US rangelands. En: Journal of Environmental Management. 111: 78-86.

Martínez A., Núñez E., Silva Y., Takahashi K., Trasmonte G., Mosquera K. \& Lagos P. 2006. Vulnerability and adaptation to climate change in the peruvian central Andes: results of a pilot study. En: Proceedings of 8 ICSHMO, Foz do Iguaço - Brazil. April 24-28. INPE. 297:305.

Recharte J., Alban L., Arévalo R., Flores E.R, Huerta L., Orellana M., Oscanoa L. \& Sánchez P. 2002. El grupo Páramo, Jalcas y Punas del Perú: Instituciones y acciones en beneficio de comunidades y ecosistemas alto andinos. En Anales de la Reunión del Grupo Internacional de Páramos. Bogotá, Colombia.

Riera P., García D., Kriström B. \& Brännlund R. 2005. Manual de economía ambiental y de los recursos naturales. Thomson. Madrid - España. 355 pp.

Sarmiento M \& Rodríguez A. 2005. Métodos de valoración ambiental: un nuevo método basado en la variación del Producto Interior Bruto. En: Catastro. Abril 2005. 59:91.

Siffredi G. 2009. Enfoques y acciones para la conservación y manejo de pastizales en Argentina. En: $5^{\circ}$ Congreso de la Asociación Argentina para el Manejo de los Pastizales Naturales. 8 pp.

Villena M \& Lafuente E. 2012. Valoración económica de bienes ambientales por beneficiarios circundantes y no circundantes. En: Cuadernos de Economía. Vol XXXI. $\mathrm{N}^{\circ} 56.33 \mathrm{pp}$. 
Whitehead J. 1990. Measuring willingness-to-pay for wetlands preservation whit the contingent valuation method. En: Wetlands, Vol 10, N 2. $187-201$.

Yahdjian L. \& Sala O. 2011. El futuro de los pastizales sudamericanos. En: Interciencia. Vol $36 \mathrm{~N}^{\circ} 2.153: 158$.
Zarria M \& Flores E.R. 2012. Condición ecológica y manejo de pastizales en los sistemas de producción de alpacas. En: VI Congreso Mundial de Camélidos Sudamericanos. Arica 21 - 23 Noviembre.

${ }^{1}$ Investigador Asociado. Laboratorio de Utilización de Pastizales del Departamento de Producción Animal. Universidad Nacional Agraria La Molina. Avenida La Molina s/n - La Molina, Apdo. 12-056 - Lima 12- Perú. cbarrantes@lamolina.edu.pe.

${ }^{2}$ Profesor de Manejo de Pastizales. Laboratorio de Utilización de Pastizales del Departamento de Producción Animal. Universidad Nacional Agraria La Molina. Avenida La Molina s/n - La Molina, Apdo. 12-056 - Lima 12- Perú. efm@lamolina.edu.pe. 